\title{
Las adicciones no relacionadas a sustancias (DSM-5, APA, 2013): un primer paso hacia la inclusión de las Adicciones Conductuales en las clasificaciones categoriales vigentes.
}

Non-substance related addictions (DSM-5, APA 2013): A first step towards the inclusión of Behavioral Addictions in current categorical classifications.

\author{
Alfredo H. Cía ${ }^{1}$
}

\section{RESUMEN}

Se evalúan los cambios en la nomenclatura de las Adicciones en el DSM-5 y la inclusión de una nueva categoría dentro de las mismas, a la que denomina "Trastornos no relacionados a sustancias" y con la cual se designa a las llamadas adicciones conductuales. Dentro ella se incluye incluye como única patología aprobada al juego patológico o ludopatía con un nuevo rótulo: "Trastorno por juego de apuestas". El inconveniente de tal etiqueta diagnóstica para su aceptación y uso por el público en general es que puede dar lugar al supuesto erróneo de que la práctica del juego genera siempre enfermedad. Por otro lado, sin embargo, el considerar al juego patológico una adicción y no un trastorno del control de impulsos ha sido un gran avance. Lamentablemente no fue incluida la "Adicción a Internet" en el nuevo rubro, pese a su enorme y creciente importancia mundial. Se describen además las características clínicas de esta última enfermedad.

PALABRAS CLAVE: Diagnóstico, adicciones conductuales y a Internet, adicción al juego de apuestas, DSM-5.

\section{SUMMARY}

Changes in DSM-5 nomenclature of Addictions are evaluated and commented upon. Particular attention is paid to the inclusión of a new category named "Non-substance related Disorders" which designates the so-called behavioral addictions. The only entity accepted here is pathological gambling or ludopathy, using the new label "Gambling Addiction". The inconvenience of such label for its acceptance and use by the general public is that it may lead to the erroneous assumption that the practice o gambling can always cause the illness. On the other hand, however, considering pathological gambling as an addiction and not an impulse control disorder constitutes a great step forward. Regrettably "Internet Addiction" has not been included under the new rubric, in spite of its enormous and growing relevance across the world. The clinical characteristics of the latter are described.

KEY WORDS: Diagnosis, behavioral and internet addictions, gambling addiction, DSM-5.

${ }^{1}$ Presidente, Asociación Psiquiátrica de América Latina. Santo Domingo República Dominicana. 


\section{Introducción a las adicciones conductuales}

Históricamente, cuando se hablaba de adicción nos referíamos a adicción a las drogas. Sin embargo, se ha descubierto que el elemento esencial de todos los trastornos adictivos es la falta de control, es decir, el descontrol de la persona afectada sobre determinada conducta, que al comienzo le es placentera pero que luego va ganando terreno entre sus preferencias hasta llegar a dominar su vida.

En el momento actual está claro que las adicciones no se limitan a las conductas generadas por el consumo incontrolable de sustancias (por ejemplo: cannabis, anfetaminas, cocaína, opiáceos, cafeína, nicotina o alcohol), sino que existen hábitos de conducta aparentemente inofensivos que, en determinadas circunstancias, pueden convertirse en adictivos e interferir gravemente en la vida cotidiana de las personas afectadas (1-3).

Como ocurre en las adicciones químicas, las personas adictas a determinada conducta experimentan, cuando no pueden llevarla a cabo, un síndrome de abstinencia caracterizado por un profundo malestar emocional (estado de ánimo disfórico, insomnio, irritabilidad e inquietud psicomotora). Tal como ocurre en el ámbito de las drogas, es difícil que un adicto conductual se considere como tal y acepte el rótulo diagnóstico, por un tiempo prolongado.

Cuando la adicción conductual avanza, los comportamientos se vuelven automáticos, son activados por las emociones e impulsos, con pobre control cognitivo y autocrítica sobre ellos. El adicto está ávido de gratificación inmediata y no repara en las posibles consecuencias negativas de esa conducta.

El aspecto nuclear de la adicción conductual no es el tipo de conducta implicada, sino la forma de relación que el sujeto establece con ella (4). En realidad, al decir de Echeburúa, cualquier actividad normal que resulte placentera para un individuo puede convertirse en una conducta adictiva. Lo esencial del trastorno es que el enfermo pierde el control sobre la actividad elegida y continúa con ella a pesar de las consecuencias adversas de todo tipo que ella produce (5).

El comportamiento se activa por una emoción que puede oscilar desde un deseo intenso o avidez hasta una auténtica obsesión y puede generar un síndrome de abstinencia si se deja de concretarlo. El afectado, focalizado totalmente en su adicción específica, se desinteresa por las actividades de tiempo libre que antes consideraba placenteras $(6,7)$.

Desde una perspectiva conductual o de la teoría del aprendizaje todas las conductas adictivas se activan al comienzo mediante un mecanismo de refuerzo positivo como es el placer experimentado o euforia (high), pero a medida que la conducta se perpetúa, dicho reforzador termina siendo negativo y responde a la necesidad de no experimentar el malestar o abstinencia que experimenta al no llevar a cabo dicha conducta (8).

Una persona normal puede escribir un mensaje o conectarse a la red por placer o por la funcionalidad de la conducta en sí misma; por el contrario, una persona adicta lo hace para aliviar su disforia o malestar emocional (aburrimiento, soledad, rabia, o excitación) (9). En síntesis, algunas conductas normales incluso saludables- se pueden convertir en anormales según la frecuencia, intensidad o tiempo y dinero invertidos. Vale decir que una adicción sin droga es toda aquella conducta repetitiva que produce placer y alivio tensional, sobre todo en sus primeras etapas, y que lleva a una pérdida de control de la misma, perturbando severamente la vida cotidiana, a nivel familiar, laboral o social, a medida que transcurre el tiempo $(10,11)$.

Finalmente, es frecuente la combinación de una adicción conductual con una o más adicciones a sustancias. Así, por ejemplo, el juego patológico se asocia principalmente con el tabaquismo y el consumo abusivo de alcohol (12).

En resumen, si una persona pierde el control sobre una conducta placentera, que luego se destaca y sobresale del resto de actividades en su vida, se ha convertido en un adicto conductual.

Los síntomas principales de una adicción conductual son:

- Intenso deseo, ansia o necesidad imparable de concretar la actividad placentera.

- Pérdida progresiva del control sobre la misma, hasta llegar al descontrol.

- Descuido de las actividades habituales previas, tanto las familiares, como las académicas, laborales o de tiempo libre.

- Estas consecuencias negativas suelen ser advertidas por personas allegadas que se lo comunican al adicto, quien, a pesar de ello, no detiene la actividad 
y se pone a la defensiva, negando el problema que padece.

- Progresiva focalización de las relaciones, actividades e intereses en torno a la adicción, con descuido o abandono de los intereses y relaciones previos, ajenos a la conducta adictiva.

- Irritabilidad y malestar ante la imposibilidad de concretar el patrón o secuencia adictiva (abstinencia) e imposibilidad de dejar de hacerlo, pasado un corto período.

La dificultad para el afrontamiento de los problemas, la baja autoestima, la impulsividad, la no tolerancia a estímulos físicos o psicológicos desagradables como el dolor o la tristeza, un estado de ánimo depresivo o disfórico y la búsqueda de sensaciones fuertes aumentan la predisposición y la vulnerabilidad para que un sujeto desarrolle una adicción en general y, de esta índole, en particular.

El consumo de ciertas sustancias o drogas, produce en el sistema nervioso central un aumento considerable de dopamina, llevando a una sensación de euforia. El aumento de la dopamina, puede generarse además a través de otro tipo de conductas como son el juego problemático, las relaciones sexuales descontroladas o las compras compulsivas.

\section{Semejanzas entre adicciones a sustancias y adicciones conductuales}

Existe creciente evidencia que sugiere que las adicciones conductuales se asemejan a las adicciones a sustancias en muchos aspectos, incluyendo su:

- Clínica y fenomenología,

- Historia natural,

- Comorbilidad,

- Tolerancia y abstinencia,

- Aspectos genéticos superpuestos,

- Circuitos neurológicos implicados y

- Respuestas al tratamiento

Dentro de muchas adicciones conductuales, las siguientes parecen ser las más frecuentes o conocidas:

- Trastorno por Juegos de Apuestas (Ludopatía o Juego Patológico)

- Adicción a Internet y a las nuevas tecnologías virtuales.

- Compras compulsivas (Oniomanía)

- Adicción al Sexo

- Adicción al Trabajo

Nueva caracterización de las Adicciones y aparición de las adicciones conductuales en el DSM-5 (APA, 2013)

En el DSM-5 (APA, 2013) (13) se ha propuesto una nueva categoría denominada Trastornos adictivos y relacionados a sustancias

Dentro de este capítulo, se incluyen los:

- Trastornos relacionados a sustancias.

- Trastornos no relacionados a sustancias.

Esta última categoría incluye solamente la que denomina Gambling Disorder o Trastorno por Juego de Apuestas (F63.0) (La traducción es del autor, dado que aún no hay versión en castellano del Manual). De este modo, el denominado Juego Patológico por el DSM-IV-TR, APA, 2001(14) hasta hace unos meses, ha pasado a formar parte del nuevo capítulo de Adicciones del DSM-5 (13), en una nueva subcategoría denominada trastornos adictivos no relacionados a sustancias. Lamentablemente, el nuevo manual clasificatorio no menciona explícitamente que esta última subcategoría equivale a decir Adicciones Conductuales.

Por otra parte, considero que el nuevo rótulo de Trastorno por Juego (de apuestas) a secas, puede llevar a la interpretación equivocada por parte de la gente común, opinión pública y de la comunidad en general, de que la práctica de todo juego de azar o de apuestas lleva inexorablemente a padecer una enfermedad adictiva al mismo, cuando los datos epidemiológicos poblacionales han demostrado que la prevalencia de por vida de este trastorno es de un 0,5 a $1,5 \%$, en tanto que los juegos de azar son practicados por un 70 a $80 \%$ de la población general adulta, en la mayoría de los países del orbe en los cuales están permitidos legalmente.

En el nuevo trastorno por juego (F63.0) se conservaron 9 criterios descriptivos presentes en el DSM-IV-TR(14), eliminándose el de cometer diversos actos ilegales para financiar el juego, tales como robo, estafa, malversación, emisión de cheques sin fondo, etc. Se consideró que esto no era relevante para efectuar el diagnóstico del trastorno.

Como dato favorable al cambio, se ha dado un paso importante en la nueva clasificación al reconocer que los problemas derivados del juego constituyen una adicción y no son simplemente un trastorno del control de impulsos. Ha sido un acierto que se rescatara el término Adicción en el DSM-5 (13) al 
incluirse una nueva categoría o capítulo denominado Trastornos Adictivos y Relacionados a sustancias. Es igualmente positivo el que se haya descartado en el mismo capítulo, el término dependencia, utilizado en el DSM-IV-TR (14), en el cual el capítulo respectivo se denominaba Trastornos relacionados a Sustancias y cuya primera división o categoría, llamada trastorno por uso de sustancias, los términos dependencia y abuso.

Hace algunos años se había argumentado que la palabra adicción era un término peyorativo que podría contribuir a la estigmatización. El término dependencia ha sido tradicionalmente usado en medicina para describir la dependencia física que se refiere a las adaptaciones y desadaptaciones que ocurren en el organismo como resultado del uso de diversos fármacos. De otro lado, el término adicción se refiere a la pérdida de control, con intensa urgencia para buscar y recibir una droga, aunque la misma produzca consecuencias adversas.

El resultado de estas denominaciones equívocas era que los clínicos que veían evidencias de tolerancia y síntomas de abstinencia asumieran que esto significaba adicción, lo cual es totalmente equivocado. El mundo médico necesitaba un cambio drástico en la rotulación y se ha rescatado en el DSM-5 (13) la palabra adicción. De esta manera se diferencia claramente y se evita el daño que aún se inflige a los pacientes que necesitan de cierta medicación y se la han retirado porque su médico creía que eran adictos, simplemente por ser físicamente dependientes a la misma.

Por otra parte, la llamada Adicción a Internet o a las nuevas tecnologías virtuales, pese a los estudios que se han realizado y a la abundante experiencia clínica y terapéutica acumulada, con una alarmante prevalencia creciente en el mundo, sobre todo en grupo etarios infanto-juveniles en las dos últimas décadas, ya con méritos suficientes para ser incorporada al nuevo Manual, no ha sido incluida.

Otro tanto ha ocurrido con la Adicción al sexo no parafilico, que fue considerado por el respectivo grupo de trabajo en el Comité del DSM-5 (13) aunque la experiencia con el mismo era mucho menor.

Ha sido solamente en la Sección III del DSM-5, Condiciones para más estudios en el futuro (13) que se ha incluido el llamado Internet gaming disorder o Trastorno por juegos de internet. Se le describe como aquellos juegos virtuales que ocurren típicamente en red y consumen al usuario un promedio 8 a 10 horas diarias (se aclara que esta categoría en estudio no incluye a los juegos de apuestas por internet) y que presenta una prevalencia elevada en niños y adolescentes, sobre todo en países de Oriente, como China y Corea del Sur. Su prevalencia ha sido estimada en $8,4 \%$ en varones y $4,5 \%$ en mujeres (13). Se aclara asimismo que el uso excesivo de Facebook o de pornografía online no son consideradas análogos a este trastorno, y que se necesitan futuros estudios para su inclusión como adicciones (13).

\section{Descripción de dos de las Adicciones Conductuales más relevantes}

\section{Trastorno por Juego de Apuestas (13) o Ludopatía} (15):

La práctica de los juegos de azar está muy presente en nuestra sociedad. La facilidad para acceder al juego, la inmediatez de la recompensa y la falsa sensación de control del sujeto sobre sus resultados hacen que el juego suponga una conducta con riesgo de transformarse en adictiva.

Existen diferentes motivos por los que un sujeto mantiene la conducta: olvidar los problemas, obtener ganancias, compensación de la ausencia de relaciones sociales, la ilusión de control, etc. Esta pérdida de control en el jugador, genera sensación de ansiedad y depresión que pueden llevarle a poner en riesgo su vida, por pensamientos y conductas suicidas en la fase de desesperación y desesperanza, en etapas avanzadas de la ludopatía.

\section{Criterios diagnósticos de Gambling Disorder o Trastorno por Juego de apuestas (F63.0) del DSM- 5.}

A- Conducta problemática de juego de apuestas recurrente y persistente, que lleva a incapacidad o distrés clínicamente significativos, como sucede cuando el individuo exhibe 4 (o más) de los siguientes síntomas durante un período de 12 meses:

1.- Necesidad de jugar cantidades crecientes de dinero para lograr la excitación deseada.

2.- Está inquieto o irritable cuando intenta interrumpir o detener el juego.

3.- Ha efectuado reiterados esfuerzos no exitosos para controlar, interrumpir o detener el juego.

4.- Está frecuentemente preocupado por el juego de apuestas (Ej.: pensamientos persistentes de revivir experiencias pasadas, planear la próxima sesión, pensar maneras de obtener el dinero para apostar). 
5.- Juega frecuentemente cuando se siente con distrés (ej: desesperanzado, culpable, ansioso o deprimido).

6.- Después de perder dinero en el juego, vuelve con frecuencia otro día para intentar recuperarlo (rescatar o cazar (chasing) las propias pérdidas).

7.- Miente para ocultar su grado de implicación en el juego.

8.- Ha arriesgado o perdido relaciones interpersonales significativas, oportunidades de trabajo y educativas o profesionales debido al juego.

9.- Confía en que los demás le proporcionen el dinero que alivie la desesperada situación financiera causada por el juego.

B- La conducta de juego de apuestas no puede ser atribuible a un episodio maníaco.

\section{Especificadores:}

- Episódico: Reúne los criterios diagnósticos y luego pueden pasar meses sin síntomas.

- Persistente: los síntomas son continuos y persisten por años.

- En remisión temprana: Luego de haber sido diagnosticado, no reúne luego ninguno de los criterios durante un periodo de entre 3 a 12 meses.

- En remisión sostenida: similar al anterior, pero luego asintomático por mas de 12 meses.

- Nivel de severidad:

- Leve (reúne 4 a 5 de los 9 criterios)

- Moderado (reúne de 6 a 7 criterios )

- Severo (reúne de 8 a 9 criterios)

A priori, podría afirmarse que con las nuevas definiciones acerca de los criterios mínimos para llegar a un diagnóstico, que son solo 4 de 9 , en lugar de reunir 5 de 10, como era en el DSM-IV-TR (14), los porcentajes de prevalencia se elevarán algo en la población.

Trastorno por Juegos de Internet (Internet gaming disorder). En la sección de condiciones que requieren mayores estudios se ha incluido este trastorno. Los criterios propuestos para su diagnóstico son los siguientes:

1.- Preocupación por los juegos por Internet, que pasan a ser la actividad dominante.

2.- Síntomas de abstinencia cuando se le impide jugar ( irritabilidad, ansiedad o tristeza)

3.- Tolerancia: se necesitan cantidades mayores de tiempo para practicarlos.

4.- Intentos fracasados para controlar la participación en los juegos de Internet.

5.- Pérdida de interés en otros hobbies o aficiones y en otras formas de entretenimiento.
6.- Uso continuo de los juegos de Internet a pesar de conocer los problemas psicosociales que generan. 7.- Ha engañado a familiares, terapeutas y otros respecto a la cantidad de juego por Internet que practica.

8.- Uso de los juegos desarrollados, aunque su prevalencia creciente es universal por Internet para escapar o aliviar un humor negativo (por ejemplo, sentimientos de desesperanza, culpa o ansiedad)

9.- Ha descuidado o perdido relaciones significativas, su empleo u oportunidades educativas o laborales debido a su participación en juegos por Internet.

\section{Nota:}

Este trastorno es distinto al Juego de apuestas por Internet, que se incluye dentro del Trastorno por Juegos de Apuestas.

Entre otras características, se menciona que los juegos por Internet ocurren típicamente en red, con otros participantes o competidores y un promedio 8 a 10 horas diarias, presentando una prevalencia elevada en niños y adolescentes, sobre todo de países orientales, aunque su presencia a nivel mundial es creciente. Se aclara igualmente que el uso excesivo de Facebook o de pornografía online no son consideradas análogos a este trastorno, y que se necesitan futuros estudios para su inclusión como adicciones conductuales.

\section{Adicción a Internet}

Llama la atención la no inclusión en el Manual de la Adicción a Internet y a las nuevas tecnologías virtuales en su conjunto, con las múltiples variantes actuales, dada la amplia experiencia clínica, investigaciones y comprobaciones acerca de su existencia como entidad a lo largo de casi tres décadas.

El uso de internet está cada vez mas difundido en el mundo globalizado en el que vivimos. Aunque esta herramienta en un principio nos permite hacer mejoras en nuestro día a día (recabar información, mantener el contacto a través del correo electrónico, etc.) su utilización con fines recreativos excesiva y persistente puede conducir a una adicción. De hecho, su importancia clínica y epidemiológica la sitúa como una de las patologías emergentes de mayor impacto en el siglo actual.

La persona que sufre adicción a internet, se caracteriza por tiempos de conexión muy prolongados con fines recreativos a lo largo de cada día, que 
pueden llevarle a descuidar otras áreas importantes de su vida como el trabajo, las relaciones sociales, la alimentación, el descanso, etc.

Existen una serie de antecedentes que actúan como factores de riesgo de esta adicción: introversión, depresión, baja autoestima, búsqueda de sensaciones, ansiedad social y timidez. Los sujetos más proclives a sufrir problemas de este tipo son las personas que presentan déficits específicos en sus habilidades de relación y comunicación.

Los adictos a internet experimentan síntomas ansiosos, irritabilidad, desajuste emocional y problemas en la interacción social. La mayoría admite que tecleando frente a la pantalla se expresa mucho mejor que con una comunicación verbal presencial. A veces crean un personaje idealizado a su medida. La adicción a las actividades en línea incluye el chat, la navegación en la web, los juegos de azar online, los juegos en red, el relacionamiento social virtual, o la búsqueda compulsiva de sexo, juegos violentos o pornografía.

Los problemas con Internet surgen cuando sus usuarios recreativos descuidan sus rutinas diarias para permanecer conectados, o bien sustraen horas al sueño nocturno, invirtiendo el ritmo circadiano (se levantan al mediodía y permanecen conectados hasta el amanecer). Algunos afectados, además de reducir el número de horas de sueño o actividades, llegan a eliminar comidas. Permanecer conectados más de 3 a 4 horas diarias facilita el aislamiento de la realidad, el desinterés por otros temas, el bajo rendimiento académico u laboral, los trastornos de conducta, así como el sedentarismo y la obesidad o descuidos para alimentarse.

Hay personas más vulnerables a padecer esta adicción. A nivel demográfico, los adolescentes constituyen un grupo de riesgo para esta adicción porque tienden a buscar sensaciones nuevas y son los que más se conectan a Internet, además de estar más familiarizados con las nuevas tecnologías (16). Sin embargo, a pesar de que la disponibilidad actual de las nuevas tecnologías en las sociedades desarrolladas de Oriente y Occidente es muy amplia, sólo un reducido número de personas muestran problemas de adicción (17).

En algunos casos hay ciertas características de personalidad o estados emocionales que aumentan la vulnerabilidad psicológica a las adicciones: la impulsividad; la disforia, la intolerancia a los estímulos displacenteros, tanto físicos (dolores, insomnio o fatiga) como psíquicos (disgustos, preocupaciones o responsabilidades) y la búsqueda exagerada de emociones fuertes. Sin embargo, a veces subyace a la adicción un problema de personalidad -timidez excesiva, baja autoestima o rechazo de la imagen corporal, por ejemplo- o un estilo de afrontamiento inadecuado ante las dificultades cotidianas. A su vez, los problemas psiquiátricos previos (depresión, TDAH, trastorno de ansiedad social u hostilidad) aumentan el riesgo de "engancharse" a Internet $(18,19)$.

Otras veces, se trata de personas que insatisfechos con su vida o con carencias vinculares o afectivas, intentan llenar esa falta con adicciones a sustancias o mediante conductas adictivas como las que estamos comentando (descontrol para gastos y compras, juego, uso de internet o dispositivos móviles).

En resumen, un sujeto vulnerable corre el riesgo de volverse adicto si comprueba que puede obtener recompensas inmediatas, si cuenta con algún tipo de adicción disponible que lo complazca particularmente. El estar sometido a circunstancias adversas en lo cotidiano (fracaso escolar, frustraciones afectivas, bullying, o competencia desmedida); el ser impulsado por el grupo de pares con quienes se identifica, o una sensación de vacío existencial (aislamiento social o falta de objetivos) son todos factores que pueden desencadenar el cuadro adictivo. De este modo, más que de perfil de adicto a las nuevas tecnologías, hay que hablar de una persona propensa a sufrir adicciones de cualquier tipo.

\section{Señales de alarma.}

Las principales señales de alarma que denotan una adicción a Internet o a las redes sociales y que pueden ser un reflejo de la conversión de una afición en una adicción son las siguientes $(20,21)$ :

a. Privarse de sueño ( $<5$ horas) para estar conectado a la red, a la que se dedica tiempos de conexión anormalmente altos.

b. Descuidar otras actividades importantes como el contacto y el compartir con la familia, las relaciones sociales, el estudio o el cuidado de la salud.

c. Recibir quejas en relación con el uso de la red de alguien cercano, como los padres o los hermanos, sin prestarles atención o negando el uso desmedido.

d. Pensar en la red constantemente, incluso cuando no se está conectado a ella y sentirse irritado excesivamente 
cuando la conexión falla o resulta muy lenta.

e. Intentar limitar el tiempo de conexión, pero sin conseguirlo, y perder la noción del tiempo.

f. Mentir sobre el tiempo real que se está conectado o manejando un videojuego.

g. Aislarse socialmente, mostrarse irritable y bajar el rendimiento escolar o laboral.

h. Sentir euforia y activación anómalas cuando se está delante de la computadora.

De este modo, conectarse al ordenador al llegar a casa, o al levantarse y ser lo último que se hace antes de acostarse, así como reducir el tiempo de las tareas cotidianas tales como comer, dormir, estudiar o charlar con la familia, configuran el perfil de un adicto a Internet. Más que el número de horas conectado a la red, lo determinante es el grado de interferencia y alteración en la vida cotidiana.

En definitiva, la dependencia a Internet o a las redes sociales está ya instalada cuando: hay un uso excesivo asociado a una pérdida de control, aparecen síntomas de abstinencia (ansiedad, depresión, irritabilidad) ante la imposibilidad temporal de acceder a la red, se establece la tolerancia (es decir, la necesidad creciente de aumentar el tiempo de conexión a Internet para sentirse satisfecho) y se producen repercusiones negativas en la vida cotidiana con reducción de la actividad física. El sujeto muestra ansia desmedida por las redes sociales y se produce un flujo de transrealidad que recuerda la experiencia con drogas (22).

\section{Tratamiento}

En la mayoría de los casos, las personas que padecen alguna adicción no siguen ningún tratamiento; sin embargo, existen pruebas que confirman que las personas que piden ayuda consiguen mejorar su calidad de vida de manera importante. En la mayoría de los casos, las adicciones se abordan desde una perspectiva multidisciplinaria, siendo la terapia psicológica un punto imprescindible y fundamental en la intervención.

En cualquier tratamiento, ha de tenerse en cuenta que cada individuo es único y por ello el plan de ayuda será personalizado en cada caso, buscándose siempre ayudarle a restablecer y mejorar de manera sustancial cada una de las áreas importantes de su vida: salud física y mental, relaciones familiares y sociales, trabajo, etc.
El profesional, siempre con la colaboración del paciente, trabajará sobre diferentes planos todos igualmente importantes: autoestima, control de impulsos, manejo de las emociones, estrategias de afrontamiento, herramientas para prevenir recaídas, etc. Para que el tratamiento resulte lo más eficaz posible, la intervención no solo se centrará en el propio sujeto sino que deberá fomentar la presencia de personas de su entorno, como familiares, pareja, amigos, etc. que deberán colaborar decisivamente en el proceso terapéutico.

\section{CONCLUSIONES}

La descripción inicial de "Adicciones no relacionadas a sustancias" en el DSM-5 ha sido un importante paso inicial para incorporar gradualmente las denominadas adicciones conductuales a las clasificaciones categoriales actuales. Dada su importancia creciente en lo que va del siglo, esta inclusión marcará un hito para que la salud mental mundial le otorgue la importancia que se merece y continúe, bajo este estímulo, desarrollando nuevas técnicas y recursos multidisciplinarios para su mejor diagnóstico y un tratamiento integral.

\section{Correspondencia:}

\section{Alfredo H. Cía}

Avda Santa Fé 3946, 1ro A, CP 1425,Ciudad Autónoma de Buenos Aires, Argentina.

Tel.: 5411-48313192 o 48335363

Correo electrónico: alfredocia@gmail.com

\section{REFERENCIAS BIBLIOGRAFICAS}

1. Echeburúa E, Corral P. Adicción a las nuevas tecnologías y a las redes sociales. Adicciones. 2010; 22, 91-96.

2. Echeburúa E. ¿Adicciones sin drogas? Las nuevas adicciones. Bilbao: Desclee de Brouwer; 1999.

3. Griffiths M. Does Internet and computer "addiction" exist? Some case study evidence. Ciberpsychology \& Behavior. 2000;3: 211-218.

4. Alonso-Fernández, F. Las otras drogas. Madrid: Temas de Hoy, 1996.

5. Echeburúa E, Fernandez-Montalvo J. Adicciones sin drogas. En: Perez de los Cobos JC, Valderrama JC, Cervera G, Rubio G. (Eds.). Tratado SET de trastornos adictivos. Madrid: Panamericana; 2006.p.471-476.

6. Choliz M, Echeburua E, Labrador FJ. Technological addictions: Are these the new addictions? Current Psychiatric Reviews. 2012;8: 290-291. 
7. Gossop M. Relapse and addictive behaviour. London: Routledge; 1989.

8. Marks I. Behavioural (non-chemical) addictions. British Journal of Addictions. 1990; 85: 1389-1394.

9. Potenza M. Should addictive disorders include nonsubstance related conditions? Addiction. 2006; 10 (S1): 142-151.

10. Fairburn C. La superación de los atracones de comida. Barcelona: Paidos; 1998.

11. Labrador FJ, Villadangos S. Adicciones a nuevas tecnologías en jóvenes y adolescentes. En: Echeburúa E, Labrador FJ, Becoña E. (Eds.). Adicción a las nuevas tecnologías en adolescentes y jóvenes.Madrid: Pirámide; 2009.p.205-220.

12. Echeburúa E, Corral P, Amor PJ. El reto de las nuevas adicciones: objetivos terapéuticos y vías de intervención. Psicología Conductual. 2005; 13: 511525.

13. American Psychiatric Association. Diagnostic and Statistical Manual of Mental Disorders, $5^{\text {th }}$. Edition (DSM-5). Washington, DC.: American Psychiatric Association; 2013.

14. American Psychiatric Association. Diagnostic and Statistical Manual of Mental Disorders, $4^{\text {th }}$. Ed., Text Revised. Washington, DC: American Psychiatric Association; 2001.

15. Organizaciòn Mundial de la salud. Clasificación Internacional de Enfermedades (OMS) CIE-10. Descripciones Clínicas y Pautas para el Diagnóstico. Madrid: Meditor, 1992.

16. Sánchez-Carbonell X, Beranuy $M$, Castellana $M$, Chamarro A, Oberst U. La adicción a Internet y al móvil: ¿moda o trastorno? Adicciones. 2008; 20: 149-160.

17. Becoña E. Adicción a nuevas tecnologías. Vigo: Nova Galícia Edicións; 2006.

18. Estévez L, Bayón C, De la Cruz J, Fernández-Liria A. Uso y abuso de Internet en adolescentes. En: Echeburúa E, Labrador FJ, Becoña E. (eds.), Adicción a las nuevas tecnologías en adolescentes y jóvenes. Madrid: Pirámide; 2009.p.101-128.

19. Yang CK, Choe BM, Balty M, Lee JH. SCL-90-R and 16 PF profiles of senior high school students with excessive Internet use. Canadian Journal of Psychiatry. 2005; 50: 407-414.

20. Young K. Internet Addiction: The emergence of a new clinical disorder. Cyberpsychology \& Behavior. 1998; 1: 237-244.

21. Davis RA. A cognitive-behavioral model of pathological Internet use. Computers in Human Behavior. 2001; 17: 187-195.

22. Greenfield DN. Tratamiento psicológico de la adicción a Internet y a las nuevas tecnologías. En Echeburúa E,.Labrador FJ, Becoña E. (eds.), Adicción a las nuevas tecnologías en adolescentes y jóvenes. Madrid: Pirámide, 2009.p.189-200.

Recibido: $27 / 10 / 2013$

Aceptado: 24/11/2013 